\title{
Combined Enzyme Defect of Mitochondrial Fatty Acid Oxidation
}

\author{
Sandra Jackson, Rajinder Singh Kler, Kim Bartlett, Helen Briggs, Laurence A. Bindoff, \\ Morteza Pourfarzam, David Gardner-Medwin, and Douglass M. Turnbull \\ Division of Clinical Neuroscience and Department of Child Health, The Medical School, University of Newcastle upon Tyne, \\ Newcastle upon Tyne, NE2 4HH, United Kingdom
}

\begin{abstract}
A young girl presented with recurrent episodes of muscle weakness culminating in a severe attack of generalized muscle weakness. In the muscle mitochondria from the patient there was an abnormal pattern of intermediates of $\beta$-oxidation with an accumulation of 3-hydroxyacyl- and 2-enoyl-CoA and carnitine esters, and 3-oxoacylcarnitines. There was low activity of longchain 3-hydroxyacyl-CoA dehydrogenase in mitochondria from all tissues. The activity of long-chain 2-enoyl-CoA hydratase was low in muscle mitochondria and 3-oxoacyl-CoA thiolase activity measured with 3-oxohexadecanoyl-CoA as substrate was low in fibroblast, muscle, and cardiac mitochondria but only partial deficiency was present when the activity was measured with 3-oxooctanoyl-CoA. The activity of the long-chain 3-hydroxyacyl-CoA dehydrogenase and long-chain 3-oxoacylCoA thiolase in fibroblasts from the patient's parents was intermediate between those of controls and the patient. The patient has a combined defect of the long-chain 3-hydroxyacylCoA dehydrogenase, long-chain 3-oxoacyl-CoA thiolase, and long-chain 2-enoyl-CoA hydratase which appears to be inherited in an autosomal recessive manner. This suggests there is a multifunctional enzyme catalyzing these activities in human mitochondria and that this enzyme is deficient in our patient. (J. Clin. Invest. 1992. 90:1219-1225.) Key words: deficiency • fatty acids • mitochondria • oxidation
\end{abstract}

\section{Introduction}

Fatty acids are important metabolic fuels particularly at times of stress or fasting. They are predominantly metabolized in mitochondria, by a process requiring activation, transport into the mitochondria, and subsequent conversion to acetyl-CoA by $\beta$-oxidation. This sequence of events may be impaired by genetic or environmental factors, and over the past decade an increasing number of patients have been identified with defects of mitochondrial fatty acid oxidation. The clinical features in these patients include hypoketotic hypoglycemic coma, sudden infant death syndrome, Reye-like episodes, muscle weakness, and cardiomyopathy (1).

Address reprint requests to Professor D. M. Turnbull, Division of Clinical Neuroscience, The Medical School, University of Newcastle upon Tyne, Framlington Place, Newcastle upon Tyne NE2 4HH, United Kingdom.

Received for publication 21 January 1992 and in revised form 1 May 1992.

J. Clin. Invest.

(c) The American Society for Clinical Investigation, Inc.

$0021-9738 / 92 / 10 / 1219 / 07 \quad \$ 2.00$

Volume 90, October 1992, 1219-1225
The first step of mitochondrial fatty acid oxidation involves the activation of fatty acids to their acyl-CoA esters; these are transported into the matrix by the concerted action of carnitine palmitoyltransferase I, carnitine/acylcarnitine translocase, and carnitine palmitoyltransferase II. The fatty acyl-CoA esters undergo $\beta$-oxidation to generate acetyl-CoA. There are two or more enzymes with overlapping substrate specificities for each of the four reactions of $\beta$-oxidation. Thus, there were thought to be three acyl-CoA dehydrogenases (short-chain, mediumchain, and long-chain), two enoyl-CoA hydratases (shortchain and long-chain), two 3-hydroxyacyl-CoA dehydrogenases (short-chain and long-chain), and two 3-oxoacyl-CoA thiolases (acetoacetyl-CoA specific and a general 3-oxoacylCoA thiolase) (2). Defects of several enzymes of $\beta$-oxidation have been described including abnormalities of short-chain, medium-chain, and long-chain acyl-CoA dehydrogenases (3), electron transfer flavoprotein, electron transfer flavoprotein dehydrogenase (4), and long-chain 3-hydroxyacyl-CoA dehydrogenase (5).

The diagnosis of defects of fatty acid oxidation is not straightforward. Measurement of metabolic fuels and intermediary metabolites may be helpful because patients with defects often demonstrate a poor ketotic response and hypoglycaemia when fasted or stressed (6). The identification of abnormal organic acids, acylglycines, and acylcarnitines in the urine may be diagnostic. More recently, molecular techniques have been successfully employed in the diagnosis of medium-chain acylCoA dehydrogenase deficiency $(7,8)$. These investigations are not, however, always reliable or universally applicable and confirmation of the defect usually requires direct measurement of enzyme activity. This itself is technically difficult in that many of the substrates for these enzymes are not commercially available and must first be synthesized.

We describe our investigations of a young girl who presented with recurrent episodes of muscle weakness in whom a defect of fatty acid oxidation was a possibility. Using techniques we have developed to measure the individual acyl-CoA and acylcarnitine esters generated during fatty acid oxidation, combined with the assay of the activity of all the enzymes of $\beta$-oxidation, we have identified a combined defect involving long-chain 3-hydroxyacyl-CoA dehydrogenase, long-chain 3oxoacyl-CoA thiolase, and long-chain 2-enoyl-CoA hydratase.

\section{Methods}

Case history. The patient was the youngest of three children born to healthy, nonconsanguineous parents. Her birth and perinatal progress were normal but because of muscle disease in an earlier child, serum creatine kinase activity was measured at $8 \mathrm{~d}$ of age ( $413 \mathrm{U} /$ liter; normal range $<140 \mathrm{U} /$ liter). However, at $5 \mathrm{wk}$ the serum creatine kinase activity was normal ( $112 \mathrm{U} /$ liter). At 3 mo the only abnormality detected on examination was absent tendon reflexes. She walked unsupported at $\mathbf{2 0}$ mo and was subsequently found to have a mild equinus 
deformity and toe walking. Twice at the age of $3 \mathrm{yr}$ she had minor infections which were associated with anorexia, hypotonia, and weakness. She recovered from these episodes in $24-48 \mathrm{~h}$ without any obvious after effects. At 4 yr 2 mo she was referred to one of us (Dr. Gardner-Medwin) when mild equinovarus contracture, areflexia, and extensor plantar responses were noted. Investigation at that time was refused.

She presented again aged $4 \frac{1 / 2}{2}$ yr with a 3-d history of vomiting and anorexia followed by the onset of rapidly progressive muscle weakness requiring her urgent admission to hospital. She was noted to have a weak cough, generalized muscle weakness, and areflexia. By the next morning, the weakness had progressed and she was intubated and ventilated. Biochemical studies at this time showed there was a marked increase in serum creatine kinase activity, 33,000 U/liter, falling to $17,900 \mathrm{U} /$ liter the next day. Examination of her urine revealed only small amounts of ethylmalonic, adipic, and suberic acids. Blood glucose was normal, but increased concentrations of serum lactate (4.92 $\mathrm{mM}$, normal range $<1.7 \mathrm{mM})$, pyruvate $(0.44 \mathrm{mM}$, normal range $<0.2 \mathrm{mM})$ and ammonia $(99 \mu \mathrm{M}$, normal range $<30 \mu \mathrm{M})$ were detected on day 2 of her admission. A $10 \%$ dextrose intravenous infusion was given from day 2 . Terminally, she developed severe hyponatremia ( $103 \mathrm{mM}$ ) and died $3 \mathrm{~d}$ after admission. Urgent postmortem examination was performed $15 \mathrm{~min}$ after death and samples of muscle, liver, heart, and skin were taken for further biochemical analysis. Morphological study of iliopsoas muscle showed necrosis of 5-10\% of fibers. Surprisingly, in view of the subsequent biochemical studies, lipid storage was not marked in the muscle being present in only a small proportion of the remaining fibers. There was also a predominance of the slow oxidative type I fibers ( $95 \%$ ). There was, however, marked macrovesicular and microvesicular fat storage in the liver. There was a slight increase in fibrous tissue in both the periportal and perivenous regions but no other abnormalities were seen.

The second child, a boy, is now 6 yr old and has no symptoms or signs suggestive of a similar disorder. The first child, however, died aged $2.5 \mathrm{yr}$ and probably had the same biochemical defect as the index case. This child had been well until aged 6 mo when he had an episode in which he refused feeds and became lethargic, limp, and unresponsive. On admission to the local hospital he was severely hypotonic and areflexic and responded only to pain. The serum creatine kinase activity was 3,859 U/liter but fell to $149 \mathrm{U} /$ liter within a few days. When seen by one of us (Dr. Gardner-Medwin) 6 wk later, he had improved, was attempting to sit, but was still areflexic and hypotonic. Muscle biopsy 3 mo after the episode was normal except for type 1 fiber predominance and an excess of type $2 \mathrm{c}$ fibers. Total muscle carnitine concentration (4.7 $\mathrm{nmol} / \mathrm{mg}$ protein; normal range 7.0-22.7) and total serum carnitine concentration ( $15.3 \mu \mathrm{mol} /$ liter; normal range $26-70)$ were low. By 1 yr he sat unaided but remained mildly hypotonic and areflexic. By 2 yr he was not walking unaided and had developed plantiflexion contractures. There was a transient worsening of muscle weakness for a few days at 21 mo after a throat infection and again at 26 mo after an episode of diarrhea. At $2.5 \mathrm{yr}$ he was admitted to his local hospital moribund with a history of rapid deterioration after a 2-wk respiratory illness. He was in low output cardiac failure with an enlarged dilated heart and generalized weakness. Serum creatine kinase was not measured. He died and postmortem examination was refused.

Preparation of mitochondrial fractions. Muscle, liver, and heart were immediately placed in ice-cold isolation media and mitochondrial fractions were prepared as previously described $(9,10)$. The respiratory control ratios were 3.5 (mean $\pm \mathrm{SD}$ for five controls $3.6 \pm 0.62$ ), 4.4, and 3.6 for skeletal muscle, heart, and liver respectively. Control muscle samples were obtained from patients in whom no muscle disease was detected. Samples of heart and liver were obtained from other children who died with suspected or proven metabolic disease which were not defects of fatty acid oxidation. Cultured skin fibroblasts were grown in minimum essential medium (MEM) containing Earle's salts, supplemented with $10 \%$ fetal bovine serum, $0.1 \mathrm{mg} / \mathrm{ml}$ streptomycin, $100 \mathrm{U} / \mathrm{ml}$ penicillin, MEM nonessential amino acids, and MEM vita- mins. Mitochondria were prepared from fibroblasts by homogenization and differential centrifugation (11).

Measurement of flux and intermediates of $\beta$-oxidation. Flux through $\beta$-oxidation was determined in skeletal muscle and fibroblast mitochondrial fractions by incubation with $\left[\mathrm{U}-{ }^{14} \mathrm{C}\right]$ hexadecanoate and measuring the acid-soluble radioactivity $(11,12)$. The intermediates of $\beta$-oxidation in skeletal muscle mitochondria were measured by detecting the acyl-CoA and acylcarnitine esters formed when the mitochondrial fractions were incubated with $\left[\mathrm{U}-{ }^{14} \mathrm{C}\right]$ hexadecanoate. The extraction of acyl-CoA and acylcarnitine esters, and their subsequent analysis by radio-HPLC has been described in detail $(11,12)$.

Enzymes of $\beta$-oxidation. The activity of the enzymes of $\beta$-oxidation were measured in skeletal muscle, cardiac, and liver mitochondrial fractions and in fibroblast homogenates. Mitochondrial pellets were diluted with the appropriate volume of $25 \mathrm{mM}$ potassium phosphate, $0.2 \mathrm{mM}$ EDTA, pH 8.0, for the determination of acyl-CoA and 3-hydroxyacyl-CoA dehydrogenases; $25 \mathrm{mM}$ potassium phosphate, 0.2

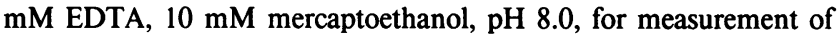
2-enoyl-CoA hydratase activity; and $100 \mathrm{mM}$ Tris, $10 \mathrm{mM}$ mercap-

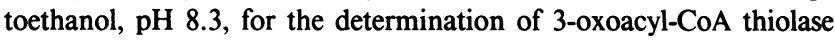
activity. The mitochondrial suspensions were sonicated on ice for 3 $\times 10 \mathrm{~s}$ with a 2 -min interval between bursts. Triton X-100 was then added to the fractions to give a Triton $X-100$ to protein ratio of $1: 1$. After incubation on ice for $30 \mathrm{~min}$, the extracts were centrifuged at $11,600 \mathrm{~g}_{\mathrm{av}}$ for $10 \mathrm{~min}$. Fibroblast homogenates were also prepared by sonication and addition of detergent (5). Enzyme activities were then measured in the supernatant as previously described $(5,13)$ except that 3-oxoacyl-CoA thiolase activity was also determined by using 3-oxooctanoyl-CoA as substrate. The protein concentration was measured by a modified Lowry procedure (14).

Immunoprecipitation of short-chain 3-hydroxyacyl-CoA dehydrogenase. Antibodies to beef heart short-chain 3-hydroxyacyl-CoA dehydrogenase were raised in rabbits (5). This antibody was added to aliquots of sonicated, detergent-solubilized, and centrifuged fibroblast homogenates or skeletal muscle, cardiac, and liver mitochondria and then incubated on ice for $2 \mathrm{~h}$. After centrifugation at $11,600 \mathrm{~g}_{\mathrm{av}}$ for $10 \mathrm{~min}$, the 3-hydroxyacyl-CoA dehydrogenase activity was assayed in the supernatant by using acetoacetyl-CoA and 3-oxohexadecanoyl-CoA as substrates. No effect on 3-hydroxyacyl-CoA dehydrogenase activity was seen when ammonium sulfate-precipitated preimmune serum from rabbit was used (5).

\section{Results}

Flux and intermediates. The rate of flux through $\beta$-oxidation was slow in both muscle (patient $4.8 \mathrm{nmol}$ of acetyl units formed per $\mathrm{min} / \mathrm{mg}$ protein; controls $10.4 \pm 1.6$ (mean \pm SD for five controls)) and fibroblast mitochondrial fractions ( 0.85 $\mathrm{nmol}$ of acetyl units formed per $\mathrm{min} / \mathrm{mg}$ protein; controls $1.72 \pm 0.04$ (mean \pm SD for three controls)).

The pattern of acyl-CoA ester intermediates was very different in the muscle mitochondria from the patient compared with controls. In normal muscle mitochondria, the only major peaks detected are those of the saturated acyl-CoA esters of chain-length $\mathrm{C}_{16}$ to $\mathrm{C}_{6}$ (Fig. $1 \mathrm{~A}$, Table I) or corresponding saturated acylcarnitine esters (Fig. $1 C$ and Table I). In mitochondria from the patient there are low concentrations of longchain saturated acyl-CoA and acylcarnitine esters but the most striking finding is the detection of additional peaks which are not seen in the control incubations (Fig. 1, $B$ and $D$ and Table I). There is accumulation of both 3-hydroxyacyl-CoA and 2enoyl-CoA ester intermediates in the incubations with skeletal muscle mitochondria from the patient. Under the chromatographic conditions used the 3-oxoacyl-CoA esters would have a 

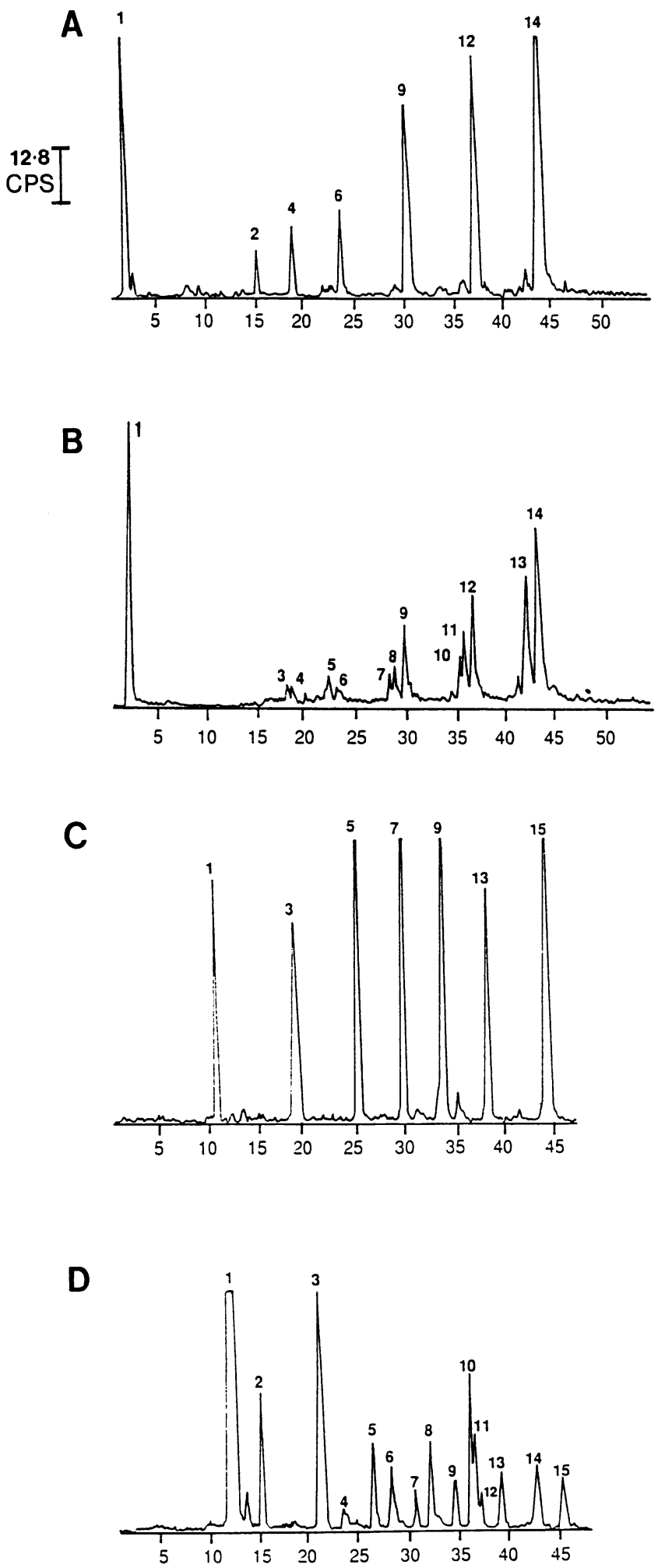

Figure 1. Radio-HPLC chromatograms of acyl-CoA and acylcarnitine esters formed after incubating skeletal muscle mitochondria with [U${ }^{14} \mathrm{C}$ ] hexadecanoate for $8 \mathrm{~min}$. Only clearly identified peaks with appropriate retention times for acyl-CoA or acylcarnitine esters are marked. The identification and amount of each individual peak are given in Table I. $(A)$ Control muscle: acyl-CoA fraction. $(B)$ Patient muscle: acyl-CoA fraction. $(C)$ Control muscle: acylcarnitine fraction. (D) Patient muscle: acylcarnitine fraction. The acyl-CoA peaks are as follows: 1 , solvent front containing TCA cycle intermediates and organic acids; 2, hexanoyl-CoA; 3, 3-hydroxydecanoyl-CoA plus retention time 5 min less than the corresponding saturated acyl-CoA ester and, therefore, a peak of 3-oxohexadecanoylCoA if present in significant amounts would be seen $\sim 1$ min after the tetradecanoyl-CoA. No such peak is detected in the mitochondrial incubations from the patient. Analysis of the acylcarnitine esters also showed the presence of additional peaks which were predominantly the 3-hydroxyacyl-carnitines (peaks 4, 6, 8, and 10 in Fig. $1 \mathrm{D}$ ). The chromatographic resolution of the 3-hydroxyacylcarnitine $\left(\mathrm{C}_{n}\right)$, 3-oxoacylcarnitine $\left(\mathrm{C}_{n}\right)$, and 2-enoylcarnitine $\left(\mathrm{C}_{n-2}\right)$ is at best partial. However, in the complex eluting at 35-38 min (Fig. $1 \mathrm{D}$ ) three peaks can be clearly discerned which co-chromatographed with chemically synthesized 3-hydroxyhexadecanoylcarnitine (peak 10), tetradec-2-enoylcarnitine (peak 11), and 3-oxohexadecanoylcarnitine (peak 12). The peaks marked 4, 6, and 8 in Fig. $1 D$ show a trailing shoulder and while the major peak is the 3-hydroxyacylcarnitine, the shoulder represents accumulation of the 2-enoylcarnitine and possibly the 3-oxoacylcarnitine esters. These findings associated with slow flux through $\beta$-oxidation are strongly suggestive of a defect of fatty acid oxidation at the 3-hydroxyacyl-CoA dehydrogenase and/or 3-oxoacyl-CoA thiolase steps. In addition, we found a high concentration of butyrylcarnitine and acetylcarnitine suggesting impaired oxidation of acetyl groups.

Activity of the $\beta$-oxidation enzymes. The activity of these enzymes was measured in mitochondrial fractions from skeletal muscle, cardiac muscle and liver, and in fibroblast homogenates ( Tables II and III). The acyl-CoA dehydrogenase activity was measured in skeletal muscle mitochondria and the activity with the butyryl-CoA, octanoyl-CoA, and hexadecanoyl-CoA was normal. 2-Enoyl-CoA hydratase activity was measured in both skeletal muscle mitochondria and fibroblast homogenates. The activity of the short-chain enzyme was normal but that of the long-chain 2-enoyl-CoA hydratase was low in muscle and in the low part of the control range in fibroblast homogenates. The activity of short-chain 3-hydroxyacyl-CoA dehydrogenase was normal but the activity of the long-chain 3-hydroxyacyl-CoA dehydrogenase was low in all tissues. In fibroblast homogenates between $20 \%$ and $35 \%$ of the 3-hydroxyacyl-CoA dehydrogenase activity using 3-oxohexadecanoyl-CoA as substrate is due to the short-chain enzyme (5). We therefore immunoprecipitated short-chain 3-hydroxyacyl-CoA dehydrogenase from both the mitochondrial fractions and fibroblast homogenates. The residual activity to the long-chain substrate was $4 \%$ of control value for skeletal muscle mitochondria, $12 \%$ for cardiac muscle mitochondria, $16 \%$ for liver mitochondria, and $6 \%$ for fibroblast homogenates after immunoprecipitation.

Mitochondrial acetoacetyl-CoA specific thiolase was mea-

Oct-2-enoyl-CoA; 4, octanoyl-CoA; 5, 3-hydroxydodecanoyl-CoA plus dec-2-enoyl-CoA; 6, decanoyl-CoA; 7, dodec-2-enoyl-CoA; 8 , 3-hydroxytetradecanoyl-CoA; 9, dodecanoyl-CoA; 10, tetradec-2enoyl-CoA; 11, 3-hydroxyhexadecanoyl-CoA; 12, tetradecanoyl-CoA; 13, hexadec-2-enoyl-CoA; 14, hexadecanoyl-CoA. The acylcarnitine peaks are as follows: 1 , acetylcarnitine; 2 , butyrylcarnitine; 3 , hexanoylcarnitine; 4, 3-hydroxydecanoylcarnitine; 5, octanoylcarnitine; 6, 3-hydroxydodecanoylcarnitine; 7, decanoylcarnitine; 8, 3-hydroxytetradecanoylcarnitine; 9 , dodecanoylcarnitine; 10, 3-hydroxyhexadecanoylcarnitine; 11 , tetradec-2-enoylcarnitine; 12, 3-oxohexadecanoylcarnitine; 13 , tetradecanoylcarnitine; 14 , hexadec-2-enoylcarnitine; 15 , hexadecanoylcarnitine. 
Table I. Acyl-CoA and Acylcarnitine Esters Detected during Incubation of Human Skeletal Muscle Mitochondria with $\left[U_{-}{ }^{14} \mathrm{C}\right]$ Hexadecanoate

\begin{tabular}{|c|c|c|}
\hline & $\begin{array}{c}\text { Control } \\
\text { (mean } \pm \text { SD) } \\
n=3\end{array}$ & Patient \\
\hline & \multicolumn{2}{|c|}{$\mathrm{nmol} / \mathrm{mg}$ protein } \\
\hline \multicolumn{3}{|l|}{ Acyl-CoA esters } \\
\hline $\begin{array}{l}\text { Solvent front containing TCA cycle } \\
\text { intermediates and organic acids }\end{array}$ & $4.9 \pm 0.08$ & 5.4 \\
\hline Hexanoyl-CoA & 0.15 & - \\
\hline \multicolumn{3}{|l|}{ 3-Hydroxydecanoyl-CoA + } \\
\hline Octanoyl-CoA & $0.13 \pm 0.08$ & 0.03 \\
\hline \multicolumn{3}{|l|}{ 3-Hydroxydodecanoyl-CoA + } \\
\hline Dec-2-enoyl-CoA & - & 0.065 \\
\hline Decanoyl-CoA & $0.17 \pm 0.06$ & 0.035 \\
\hline \multicolumn{3}{|l|}{ Dodec-2-enoyl-CoA + } \\
\hline 3-Hydroxytetradecanoyl-CoA & - & 0.097 \\
\hline Dodecanoyl-CoA & $0.47 \pm 0.17$ & 0.17 \\
\hline \multicolumn{3}{|l|}{ Tetradec-2-enoyl-CoA + } \\
\hline 3-Hydroxyhexadecanoyl-CoA & - & 0.18 \\
\hline Tetradecanoyl-CoA & $0.56 \pm 0.09$ & 0.24 \\
\hline Hexadec-2-enoyl-CoA & - & 0.21 \\
\hline Hexadecanoyl-CoA & $1.37 \pm 0.2$ & 0.37 \\
\hline \multicolumn{3}{|l|}{ Acylcarnitine esters } \\
\hline Acetylcarnitine & $13.06 \pm 3.83$ & 45.57 \\
\hline Butyrylcarnitine & - & 2.05 \\
\hline Hexanoylcarnitine & $6.87 \pm 3.2$ & 8.43 \\
\hline 3-Hydroxydecanoylcarnitine & - & 0.16 \\
\hline Octanoylcarnitine & $4.82 \pm 2.29$ & 1.66 \\
\hline 3-Hydroxydodecanoylcarnitine & - & 0.99 \\
\hline Decanoylcarnitine & $4.63 \pm 1.13$ & 0.24 \\
\hline 3-Hydoxytetradecanoylcarnitine & & 0.82 \\
\hline Dodecanoylcarnitine & $4.19 \pm 1.40$ & 0.60 \\
\hline \multicolumn{3}{|l|}{$\begin{array}{l}\text { 3-Hydroxyhexadecanoylcarnitine } \\
\text { + Tetradec-2-enoylcarnitine }\end{array}$} \\
\hline + 3-Oxohexadecanoylcarnitine & & 2.46 \\
\hline Tetradecanoylcarnitine & $2.63 \pm 0.87$ & 0.70 \\
\hline Hexadec-2-enoylcarnitine & - & 0.79 \\
\hline Hexadecanoylcarnitine & $6.72 \pm 0.34$ & 0.61 \\
\hline
\end{tabular}

The values are nanomoles of acyl-CoA or acylcarnitine ester per milligram protein after incubating mitochondria for $8 \mathrm{~min}$. The amount of TCA cycle intermediates and organic acids has been calculated assuming all products were $C_{2}$ units. When integration of separate peaks is not possible the combined amount of ester is calculated.

sured both in the presence and absence of $50 \mathrm{mM} \mathrm{KCl}$ and activity in all tissues was normal. The activity of the 3-oxoacylCoA thiolase with 3-oxohexadecanoyl-CoA as substrate was low in skeletal muscle, cardiac muscle, and fibroblasts at $10 \%$, $14 \%$, and $5 \%$ of control values, respectively. Measurement of 3-oxoacyl-CoA thiolase activity in skeletal and cardiac muscle mitochondria and fibroblast homogenates using 3-oxooctanoyl-CoA surprisingly gave very different results with the activity in the patient at $55 \%, 64 \%$, and $76 \%$ of mean control values, respectively.

The 3-oxoacyl-CoA thiolase results in liver was different from the other tissues. First, the relative activity in control liver mitochondria using the three chain length substrates $\left(\mathrm{C}_{4}, \mathrm{C}_{8}\right.$, and $\mathrm{C}_{16}$ ) was different to those seen in skeletal and cardiac muscle mitochondrial fractions, with much higher activity with 3-oxooctanoyl-CoA as substrate. The values in the liver mitochondria from the patient do not show a defect of 3-oxoacyl-CoA thiolase with the 3-oxohexadecanoyl-CoA as substrate, although the value is in the low control range.

The activity of the 2-enoyl-CoA hydratases, 3-hydroxyacylCoA dehydrogenases, and the 3-oxoacyl-CoA thiolases was measured in fibroblasts from both parents (Table III). The activity of the long-chain 2-enoyl-CoA hydratases were similar to that of the patient, and in the low part of the control range. Both parents had low activity of the long-chain 3-hydroxyacylCoA dehydrogenase with values intermediate between the patient and controls. Immunoprecipitation of the short-chain 3hydroxyacyl-CoA dehydrogenase further decreased the activity with the long-chain substrate by $45 \%$ and $50 \%$ in mother and father, respectively, compared to two controls whose values decreased by $26 \%$ and $24 \%$. The activity of the 3-oxoacyl-CoA thiolase with 3-oxohexadecanoyl-CoA as substrate was also low in both parents, the values being intermediate between those of controls and the patient.

\section{Discussion}

We describe the clinical and biochemical findings in a young girl with a defect of mitochondrial fatty acid oxidation who presented with intermittent muscle weakness. The enzyme defect in muscle was a combined deficiency of long-chain 3-hydroxyacyl-CoA dehydrogenase, long-chain 2-enoyl-CoA hydratase, and long-chain 3-oxoacyl-CoA thiolase. Deficiency of the long-chain 3-hydroxyacyl-CoA dehydrogenase was confirmed in other tissues (heart, liver, and fibroblasts) and a defect of the long-chain 3-oxoacyl-CoA thiolase was also found in heart and fibroblast mitochondria. The activity of both the long-chain 3-hydroxyacyl-CoA dehydrogenase and long-chain 3-oxoacyl-CoA thiolase in the fibroblast homogenates from the parents were intermediate between the patient and controls. This latter finding both confirms the presence of a combined defect in the patient and suggests that it is inherited in an autosomal recessive manner.

Our studies strongly suggest that the biochemical defect in this young girl is due to an abnormality in a recently isolated and characterized trifunctional enzyme of mitochondrial $\beta$-oxidation. This enzyme, which has been purified from both rat and human liver, catalyzes the 3-hydroxyacyl-CoA dehydrogenation, 2-enoyl-CoA hydration, and 3-oxoacyl-CoA thiolysis of long-chain acyl-CoA esters $(15,16)$. Unlike most other enzymes of $\beta$-oxidation it is bound to the inner mitochondrial membrane and can only be extracted by use of detergents. The enzyme from both rats and humans consists of large and small subunits ( 71 and $47 \mathrm{kD}$ in humans) that form multimeric complexes (octamer in rat, tetramer in humans).

Our studies have shown that there is low activity of all three enzymes in our patient which would suggest that the molecular defect involves both subunits. One possible explanation is that both subunits are encoded by a single gene and that this is defective in our patient. A more likely explanation is that the two subunits are encoded by separate genes, and that a mutation of one subunit causes instability of the whole multimer 
Table II. Enzyme Activities in Skeletal Muscle, Cardiac, and Liver Mitochondria

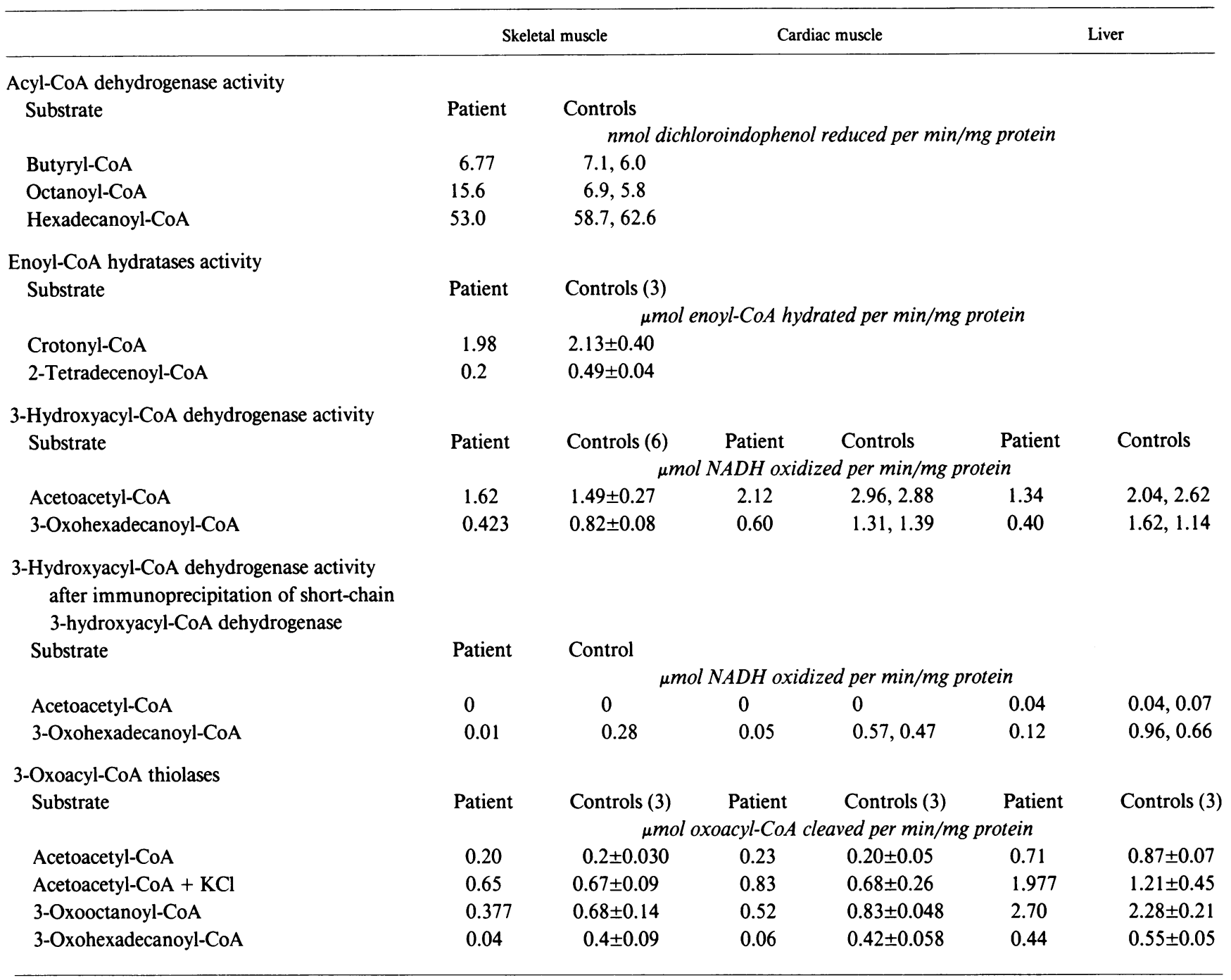

Control values are the mean \pm SD or individual values, and the number of controls is shown in parentheses.

similar to the findings in patients with mutations of the subunits of electron transfer flavoprotein (17). Clearly, the molecular basis of trifunctional enzyme deficiency will only be characterized as antibodies and cDNAs to the individual subunits become available.

Several previous patients with a defect of long-chain 3-hydroxyacyl-CoA dehydrogenase have been identified $(5,18-$ 23 ). Whereas in most of these patients the activity of the other enzymes of $\beta$-oxidation has not been measured, in the two patients in which the other enzymes were studied, the longchain 2-enoyl-CoA hydratase activity was in the low part of the control range and long-chain 3-oxoacyl-CoA thiolase activity was low at $61 \%$ and $52 \%$, respectively, of the mean control values in fibroblasts $(5)$. We postulated that there was a relationship between the three long-chain-specific enzymes and the results reported here, and the recent purification of a trifunctional enzyme of $\beta$-oxidation strongly suggests that previous cases of long-chain 3-hydroxyacyl-CoA dehydrogenase deficiency are due to a defect of the trifunctional enzyme.

The clinical features of patients reported with long-chain 3-hydroxyacyl-CoA dehydrogenase deficiency are variable, which may in part reflect different biochemical defects of the trifunctional enzyme. The age of onset of symptoms range from $3 \mathrm{~d}$ to $3 \mathrm{yr}$ and clinical manifestations have included recurrent episodes of nonketotic hypoglycaemia (5), sudden infant death $(21,23,24)$, and cardiomyopathy (19). In most cases hepatic dysfunction is a prominent feature. Two patients had a sensorimotor polyneuropathy $(20,22)$ and in one of these patients there was also a pigmentary retinopathy $(20)$. Muscle weakness has been described in several children $(5,19$, $20,22,25$ ) and has been associated with myoglobinuria (22). In our patient the muscle weakness was severe leading to respiratory failure with a very high creatinine kinase activity in serum.

In the majority of patients there has been excretion of 3-hydroxymonocarboxylic and 3-hydroxydicarboxylic organic acids $(5,19,20,23)$. This excretion may be variable and will depend upon the metabolic state of the patient (26). Urinary organic acids were not helpful in our patient, however; only two samples of urine were studied and these were obtained late in the illness. These urine samples were also analyzed for acylcarnitines by HPLC (27) and no differences were detected compared with controls. Plasma and muscle carnitine concentrations are often low in patients with $\beta$-oxidation defects, in- 
Table III. Enzyme Activities in Fibroblast Homogenates

\begin{tabular}{|c|c|c|c|c|}
\hline Substrate & Patient & Mother & Father & Controls (6) \\
\hline & \multicolumn{4}{|c|}{ nmol enoyl-CoA hydrated per $\mathrm{min} / \mathrm{mg}$ protein } \\
\hline \multicolumn{5}{|l|}{ Enoyl-CoA hydratase activity } \\
\hline Crotonyl-CoA & 108.2 & 145.1 & 146.1 & $160 \pm 42.9$ \\
\hline 2-Tetradecenoyl-CoA & 39.8 & 34.0 & 38.0 & $61.7 \pm 14.9$ \\
\hline 3-Hydroxyacyl-CoA dehydrogenase activity & \multicolumn{4}{|c|}{ nmol NADH oxidized per min/mg protein } \\
\hline Acetoacetyl-CoA & 39.9 & 43.2 & 36.8 & $42.9 \pm 8.4$ \\
\hline 3-Oxohexadecanoyl-CoA & 8.0 & 23.6 & 19.5 & $36.9 \pm 6.8$ \\
\hline \multicolumn{5}{|l|}{$\begin{array}{l}\text { 3-Hydroxyacyl-CoA dehydrogenase activity } \\
\text { after immunoprecipitation of short-chain }\end{array}$} \\
\hline 3-hydroxyacyl-CoA dehydrogenase & \multicolumn{4}{|c|}{ nmol $N A D H$ oxidized per min/mg protein } \\
\hline Acetoacetyl-CoA & 7.2 & 3.1 & 2.7 & $8.1,5.7$ \\
\hline 3-Oxohexadecanoyl-CoA & 1.8 & 12.8 & 9.8 & $31.6,32.5$ \\
\hline Thiolase activity & \multicolumn{4}{|c|}{ nmol oxoacyl-CoA cleaved per min/mg protein } \\
\hline Acetoacetyl-CoA & 10.2 & 12.4 & 11.7 & $8.8 \pm 1.2$ \\
\hline Acetoacetyl-CoA $+\mathrm{KCl}$ & 15.3 & 16.5 & 16.3 & $15.7 \pm 4.2$ \\
\hline 3-Oxooctanoyl-CoA & 10.7 & 11.9 & 9.7 & $13.9 \pm 2.2$ \\
\hline 3-Oxohexadecanoyl-CoA & 1.0 & 8.1 & 10.8 & $20.2 \pm 3.5$ \\
\hline
\end{tabular}

The control values are either the mean \pm SD and the number of controls shown in parentheses or individual values.

cluding patients with 3-hydroxyacyl-CoA dehydrogenase deficiency $(5,19,22,25)$. Although we did not measure carnitine concentrations in our patient, low concentrations were found in blood and muscle from her affected brother. The most surprising biochemical finding in patients with biochemical defects of the trifunctional enzyme is the lactic acidosis $(5,20,23$, 25 ). This is not seen in patients with other $\beta$-oxidation defects, and although the mechanism of this lactic acidosis is unknown, there are several possible explanations. We have shown that a high concentration of acetylcarnitine accumulates in the mitochondrial fractions from this patient and others with deficiency of long-chain 3-hydroxyacyl-CoA dehydrogenase (11), which suggests impaired oxidation of acetyl groups. This may be due to inhibition of either the citric acid cycle or the respiratory chain by the accumulating intermediates. Alternatively, there is the intriguing possibility that, because the trifunctional enzyme is in the inner mitochondrial membrane, its deficiency may affect respiratory chain function more directly.

Each reaction of mitochondrial $\beta$-oxidation requires two or more enzymes of different chain length specificity to enable complete oxidation of long-chain fatty acids. Our studies allow us to predict the number of enzymes catalyzing three of the four reactions of $\beta$-oxidation in human tissues. In skeletal muscle and cardiac mitochondria there was no residual 3-hydroxyacyl-CoA dehydrogenase activity with the long-chain substrate after immunoprecipitation of short-chain 3-hydroxyacyl-CoA dehydrogenase. This suggests that there are only two enzymes catalyzing the reaction in these tissues. The small amount of residual activity in fibroblast homogenates and liver is likely to be due to contamination by the peroxisomal enzyme. The long-chain 2-enoyl-CoA hydratase activity was the least affected of the three enzymes, being only decreased to $40 \%$ of control values in skeletal muscle mitochondria and in the low part of the control range in fibroblasts. In preliminary studies we have an immunoprecipitated short-chain 2-enoyl-CoA hy- dratase (antibodies raised to purified enzyme [Sigma Chemical Co., St. Louis, MO] in rabbits) from solubilized fibroblast homogenate from a control subject and the patient. These studies indicate that not all the activity towards the long-chain substrate is immunoprecipitated from the patient's mitochondria and this suggests there is an additional 2-enoyl-CoA hydratase. There were thought to be only two 3-oxoacyl-CoA thiolases in mammalian mitochondria, one of which is acetoacetyl-CoA specific and a second which acts on substrates of various chain lengths (28). Our finding that thiolysis of 3-oxohexadecanoylCoA was severely impaired whereas that of 3-oxooctanoyl-CoA was only impaired to $55 \%, 64 \%$, and $76 \%$ of mean control values in skeletal muscle mitochondria, cardiac mitochondria, and fibroblast homogenates from our patient suggests this "general" thiolase is present and that there must be three mitochondrial thiolases in these human tissues. Although there is low long-chain 3-oxoacyl-CoA thiolase activity in the muscle, heart, and fibroblasts from the patient, in liver the activity is in the low part of the normal range. This might be due to a number of factors including contamination of the liver mitochondrial fraction by peroxisomes or an additional long-chain specific 3-oxoacyl-CoA thiolase. Alternatively, the ratio of rates of thiolysis of the three different chain-length substrates in liver is different from muscle and heart, and some of the activity to the long-chain substrate might be due to overlapping substrate specificity from the medium-chain enzyme.

The history in our patient was suggestive of a $\beta$-oxidation defect, but despite her rapidly deteriorating clinical condition by the time she came under our care, she had neither a specific dicarboxylic aciduria nor the intermediary metabolite changes normally found in $\beta$-oxidation defects. This emphasizes the difficulties in making a diagnosis of a defect of mitochondrial fatty acid oxidation. We measured flux through $\beta$-oxidation and although there are many problems associated with its accurate determination in samples of tissue or cultured fibroblasts 
$(29,30)$, we measured the acid-soluble products from $\left[\mathrm{U}-{ }^{14} \mathrm{C}\right]-$ hexadecanoate oxidation which will detect defects of the longchain enzymes. Flux was low to a similar degree in both skeletal muscle and fibroblast mitochondrial fractions (46\% and $48 \%$ of control values, respectively). This degree of continued flux was surprising in view of the severity of the biochemical defect and presumably reflects that there is more overlapping enzyme activity in vivo or that the enzyme steps involved have relatively low control strength on overall pathway flux. The value of the measurement of the intermediates of $\beta$-oxidation in patients with suspected defects of this pathway is confirmed by this study. Previous studies in fibroblast mitochondria from patients with proven defects of fatty acid oxidation have shown that a characteristic pattern of intermediates is found depending upon which enzyme is defective (11). In this patient the accumulation of 3-hydroxyacyl-CoA and 3-hydroxyacylcarnitine esters first alerted us to the possibility of a defect of longchain 3-hydroxyacyl-CoA dehydrogenase. This technique has now been shown to be valuable in studying fatty acid oxidation in skeletal muscle mitochondria and it requires a relatively small amount of mitochondrial protein. We believe it will become increasingly important in the investigation of lipid storage myopathies.

\section{Acknowledgments}

We are grateful to Dr. M. A. Johnson and Dr. L. Sviland for their help with the interpretation of the morphological findings.

These investigations were supported by The Foundation for the Study of Infant Deaths, Muscular Dystrophy Group of Great Britain, Action Research for the Crippled Child, Wolfson Foundation, Newcastle University Research Committee, and Sigma-tau.

\section{References}

1. Jackson, S., and D. M. Turnbull. 1992. Lipid Disorders of Muscle. In The Molecular and Genetic Basis of Neurological Disease. R. N. Rosenberg, S. B. Prusiner, R. L. Barchi, L. M. Kunkel, and S. DiMauro, editors. Butterworths, Stoneham, MA. In press.

2. Schulz, H. 1990. Mitochondrial $\beta$-oxidation. In Fatty Acid Oxidation: Clinical, Biochemical and Molecular Aspects. K. Tanaka and P. M. Coates, editors. Alan R. Liss, Inc., New York. 23-36.

3. Roe, C. R., and P. M. Coates. Acyl-CoA dehydrogenase deficiencies. In The Metabolic Basis of Inherited Disease. C. R. Scriver, L. Beaudet, W. S. Sly, and D. Valle, editors. McGraw-Hill, Inc., New York. 889-914.

4. Loehr, J. P., S. I. Goodman, and F. E. Frerman. 1990. Glutaric acidemia type II: heterogeneity of clinical and biochemical phenotypes. Pediatr. Res. 27:311-315.

5. Jackson, S., K. Bartlett, J. Land, E. R. Moxon, R. J. Pollitt, J. V. Leonard, and D. M. Turnbull. 1991. Long-chain 3-hydroxyacyl-CoA dehydrogenase deficiency. Pediatr. Res. 29:406-411.

6. Bartlett, K., A. Aynsley-Green, J. V. Leonard, and D. M. Turnbull. 1991. Inherited disorders of mitochondrial $\beta$-oxidation. In Inborn Errors of Metabolism. Nestle Nutr. Workshop Ser. 19-40.

7. Kelly, D. P., A. J. Whelan, M. L. Ogden, R. Alpers, Z. Zhang, G. Bellus, N. Gregersen, I. Dorland, and A. W. Strauss. 1990. Molecular characterisation of inherited medium-chain acyl-CoA dehydrogenase deficiency. Proc. Natl. Acad. Sci. USA. 87:9236-9240.

8. Yokota, I., Y. Indo, P. M. Coates, and K. Tanaka. 1990. Molecular basis of medium-chain acyl-coenzyme A dehydrogenase deficiency. J. Clin. Invest. 86:1000-1003.

9. Watmough, N. J., A. K. M. J. Bhuiyan, K. Bartlett, H. S. A. Sherratt, and D. M. Turnbull. 1988. Skeletal muscle mitochondrial $\beta$-oxidation: a study of the products of the oxidation of $\left[\mathrm{U}-{ }^{14} \mathrm{C}\right]$ hexadecanoate oxidation by HPLC using continuous on-line radiochemical detection. Biochem. J. 253:541-547.

10. Birch-Machin, M. A., I. M. Shepherd, N. J. Watmough, K. Bartlett, H. S. A. Sherratt, A. Aynsley-Green, D. Milligan, V. Darley-Usmar, and D. M.
Turnbull. 1989. Fatal lactic acidosis in infancy with a defect of complex III of the respiratory chain. Pediatr. Res. 25:553-559.

11. Singh Kler, R., S. Jackson, K. Bartlett, L. A. Bindoff, S. Eaton, M. Pourfarzam, F. E. Frerman, S. I. Goodman, N. J. Watmough, and D. M. Turnbull. 1991. Quantitation of acyl-CoA and acylcarnitine esters accumulated during abnormal mitochondrial fatty acid oxidation. J. Biol. Chem. 266:22932-22938.

12. Watmough, N. J., D. M. Turnbull, H. S. A. Sherratt, and K. Bartlett. 1989. Measurement of intact acyl-CoA intermediates of $\beta$-oxidation by HPLC with on-line radiochemical and photodiode-array detection: application to the study of $\left[\mathrm{U}^{14}-\mathrm{C}\right]$ hexadecanoate oxidation by rat liver mitochondria. Biochem. J. 262:261-269.

13. Turnbull, D. M., K. Bartlett, D. L. Stevens, K. G. M. M. Alberti, G. J. Gibson, M. A. Johnson, A. J. McCulloch, and H. S. A. Sherratt. 1984. Shortchain acyl-CoA dehydrogenase deficiency associated with a lipid-storage myopathy and secondary carnitine deficiency. N. Engl. J. Med. 311:1232-1236.

14. Pedersen, G. L. 1977. A simplification of the protein assay method of Lowry et al. which is more generally applicable. Anal. Biochem. 83:346-356.

15. Uchida, Y., K. Izai, T. Orii, and T. Hashimoto. 1992. Novel fatty acid $\beta$-oxidation enzymes in rat liver mitochondria. II Purification and properties of enoyl-coenzyme A (CoA) hydratase/3-hydroxyacyl-CoA dehydrogenase/3-ketoacyl-CoA thiolase trifunctional protein. J. Biol. Chem. 267:1034-1041.

16. Carpenter, K., R. J. Pollitt, and B. Middleton. 1992. Human liver longchain 3-hydroxyacyl-coenzyme A dehydrogenase is a multifunctional membrane-bound beta-oxidation enzyme of mitochondria. Biochem. Biophys. Res. Commun. 183:443-448.

17. Frerman, F. E., and S. I. Goodman. 1985. Deficiency of electron transfer flavoprotein or electron transfer flavoprotein:ubiquinone octoreductase in glutaric aciduria Type II fibroblasts. Proc. Natl. Acad. Sci. USA. 82:4517-4520.

18. Hale, D. E., C. Thorpe, K. Braat, J. H. Wright, C. R. Roe, P. M. Coates, T. Hashimoto, and A. M. Glasgow. The L-3-hydroxyacyl-CoA dehydrogenase deficiency. In Fatty Acid Oxidation: Clinical, Biochemical and Molecular Aspects. K. Tanaka and P. M. Coates, editors. Alan R. Liss, Inc., New York. 503-510.

19. Rocchiccioli, F., R. J. A. Wanders, P. Aubourg, C. Vianey-Liaud, L. Ijlst, M. Fabre, N. Cartier, and P.-F. Bougneres. 1990. Deficiency of long-chain 3-hydroxyacyl-CoA dehydrogenase: a cause of lethal myopathy and cardiomyopathy in early childhood. Pediatr. Res. 28:657-662.

20. Bertini, E., M. Sabatelli, B. Garavaglia, A. B. Burlina, M. Rimoldi, E. Ricci, C. Dionisi-Vici, A. Bartuli, G. Sabetta, and S. DiDonato. 1990. Myopathy and sensory-motor polyneuropathy in long-chain 3-OH-acyl-CoA dehydrogenase deficiency. J. Neurol. Sci. 98(Suppl.):273.

21. Wanders, R. J. A., L. Ijlst, A. H. Van Gennip, C. Jakobs, J. P. De Jager, L. Dorland, F. J. Van Sprang, and M. Duran. 1990. Long-chain 3-hydroxyacyl-CoA dehydrogenase deficiency: identification of a new inborn error of mitochondrial fatty acid $\beta$-oxidation. J. Inherited Metab. Dis. 13:311-314.

22. Dionisi-Vici, C., A. B. Burlina, E. Bertini, C. Bachmann, M. R. M. Mazziotta, F. Zacchello, G. Sabetta, and D. E. Hale. 1991. Progressive neuropathy and recurrent myoglobinuria in a child with long-chain 3-hydroxyacyl-coenzyme A dehydrogenase deficiency. J. Pediatr. 118:744-746.

23. Duran, M., R. J. A. Wanders, J. P. de Jager, L. Dorland, L. Bruinvis, D. Ketting, L. Ijlst, and F. J. van Sprang. 1991. 3-Hydroxydicarboxylic aciduria due to long-chain 3-hydroxyacyl-coenzyme A dehydrogenase deficiency associated with sudden neonatal death: protective effect of medium-chain triglyceride treatment. Eur. J. Pediatr. 150:190-195.

24. Wanders, R. J. A., M. Duran, L. Iljst, J. P. de Jaeger, A. H. van Gennip, C. Jakobs, L. Dorland, F. J. van Sprang. 1989. Sudden infant death and long-chain 3-hydroxyacyl-CoA dehydrogenase. Lancet. ii:52-53.

25. Glasgow, A. M., A. G. Engel, D. M. Bier, L. W. Perry, M. Dickie, J. Todaro, D. I. Brown, and N. F. Utter. 1983. Hypoglycaemia, hepatic dysfunction, muscle weakness, cardiomyopathy, free carnitine deficiency and long-chain acylcarnitine excess responsive to medium-chain triglyceride diet. Pediatr. Res. 17:319-326.

26. Pollitt, R. J. 1990. Clinical and biochemical presentations in twenty cases of hydroxydicarboxylic aciduria. In Fatty Acid Oxidation: Clinical, Biochemical and Molecular Aspects. K. Tanaka and P. M. Coates, editors. Alan R. Liss, Inc., New York. 495-502.

27. Bhuiyan, A. K. M. J., S. Jackson, D. M. Turnbull, A. Aynsley-Green, J. V. Leonard, and K. Bartlett. 1992. The measurement of carnitine and acyl-carnitines: application to the investigation of patients with suspected inherited disorders of mitochondrial fatty acid oxidation. Clin. Chim. Acta. 207:185-204.

28. Staak, H., J. F. Binstock, and H. Schulz. 1978. Purification and properties of a pig heart thiolase with broad chain length specificity and comparison of thiolases from pig heart and Escherischia coli. J. Biol. Chem. 253:1827-1831.

29. Veerkamp, J. H., H. T. B. Van Moerkerk, J. F. C. Glatz, J. G. E. M. Zuurveld, A. E. M. Jacobs, and J. M. Wagenmakers. $1986 .{ }^{14} \mathrm{CO}_{2}$ production is no measure of $\left[{ }^{14} \mathrm{C}\right]$ fatty acid oxidation. Biochem. Med. Metab. Biol. 35:248-59.

30. Sherratt, H. S. A., N. J. Watmough, M. A. Johnson, and D. M. Turnbull. 1988. Methods for study of normal and abnormal skeletal muscle mitochondria. Methods Biochem. Anal. 33:243-335. 